PROCEEDINGS OF THE

AMERICAN MATHEMATICAL SOCIETY

Volume 131, Number 3, Pages 665-673

S 0002-9939(02)06798-9

Article electronically published on October 15, 2002

\title{
QUANTUM AUTOMORPHISM GROUPS OF FINITE GRAPHS
}

\author{
JULIEN BICHON
}

(Communicated by David R. Larson)

\begin{abstract}
A quantum analogue of the automorphism group of a finite graph is introduced. These are quantum subgroups of the quantum permutation groups defined by Wang. The quantum automorphism group is a stronger invariant for finite graphs than the usual automorphism group. We get a quantum dihedral group $D_{4}$.
\end{abstract}

\section{INTRODUCTION}

The problem of defining the quantum automorphism group of a quantum space arises quite naturally in noncommutative geometry [3, 5]. In his paper $[8$, S. Wang solves this problem for the finite space $X_{n}$ with $n$ points. The main step was to formulate the universal problem that the quantum automorphism group must solve. The constructed object $A_{a u t}\left(X_{n}\right)$ is a compact quantum group in the sense of Woronowicz [9] and is called "the quantum permutation group on $n$ symbols". Loosely speaking $A_{\text {aut }}\left(X_{n}\right)$ is the $C^{*}$-algebra of functions on the usual permutation group where the commutativity relations have been forgotten (and is infinitedimensional for $n \geq 4$ ). Wang also discusses the quantum automorphism groups of noncommutative finite-dimensional $C^{*}$-algebras (see the remark at the end of section 2). The representation theory of $A_{\text {aut }}\left(X_{n}\right)$ has been described recently by T. Banica [2]: the irreducible representations of $A_{\text {aut }}\left(X_{n}\right)$ have the same fusion rules as the ones of $S O(3)$ (if $n \geq 4$ ).

In this paper we discuss the quantum automorphism groups of finite graphs. These are quantum subgroups of the quantum permutation groups. Our results clearly illustrate the richness of Wang's principle for quantum automorphism groups. For example let us consider the graph with $n$ vertices and without edges: the quantum automorphism group is obviously the quantum permutation group. Now let us consider the complete graph with $n$ vertices where every pair of vertices is an edge: the quantum automorphism group is the usual permutation group. This example shows that the construction of the quantum automorphism group is far more involved than a simple freeness procedure from the usual automorphism group to the quantum one. It also shows that the quantum automorphism group is a stronger invariant for finite graphs than the usual one.

For a special graph with 4 vertices and 4 edges (which is not a square) we obtain a quantum dihedral group $D_{4}$ whose $C^{*}$-algebra is infinite-dimensional.

Received by the editors December 23, 1998 and, in revised form, March 21, 2000.

2000 Mathematics Subject Classification. Primary 16W30, 46L87.

(C)2002 American Mathematical Society 
Our work is organized as follows. In section 2 we recall some basic definitions and results on compact quantum groups and quantum automorphism groups and in section 3 we describe the quantum automorphism groups of finite graphs.

\section{Compact quantum groups and quantum Automorphism Groups}

We first recall some basic notions and results which will be used freely in the rest of the paper.

Let us recall that a compact quantum group 9, 11 is a pair $(A, \Delta)$ where $A$ is a $C^{*}$-algebra (with unit) and $\Delta: A \longrightarrow A \otimes A$ is a coassociative $*$-homomorphism such that the sets $\Delta(A)(A \otimes 1)$ and $\Delta(A)(1 \otimes A)$ are both dense in $A \otimes A$ (where $\otimes$ stands for the minimal $C^{*}$-tensor product). By abuse of notation a compact quantum group is often identified with its underlying $C^{*}$-algebra. A morphism between compact quantum groups $A$ and $B$ is a $*$-homomorphism $\pi: A \longrightarrow B$ such that $\Delta \circ \pi=(\pi \otimes \pi) \circ \Delta([\mathbf{7})$.

Given a compact quantum group $A$, there is a canonically defined Hopf $*$-algebra $A^{o}$ (which we call the algebra of representative functions) which is dense in $A$. The existence of the Haar measure on $A$ [6] 11] shows that furthermore $A^{o}$ is unitarizable [9: for every matrix $u=\left(u_{i j}\right) \in M_{n}(A)$ such that $\Delta\left(u_{i j}\right)=$ $\sum u_{i k} \otimes u_{k j}$ and $\varepsilon\left(u_{i j}\right)=\delta_{i j}$, there exists a matrix $F \in G L_{n}(\mathbb{C})$ such that the matrix $F u F^{-1}$ is unitary (in other words every $A^{\circ}$-comodule is unitarizable). If $\pi: A \longrightarrow B$ is a morphism of compact quantum groups, then $\pi\left(A^{\circ}\right) \subset B^{\circ}$ and $\pi$ is a Hopf algebra morphism ([7]).

Conversely if $A^{o}$ is a unitarizable Hopf *-algebra (a CQG algebra in [4]), the upperbound of $C^{*}$-semi-norms exists on $A^{o}$ (since $A^{o}$ is generated by the entries of unitary matrices) and is a $C^{*}$-norm (use the regular representation; see [4, 4.4). Let $C^{*}\left(A^{o}\right)$ be the enveloping $C^{*}$-algebra of $A^{o}$. Then $C^{*}\left(A^{o}\right)$, endowed with the obvious coproduct, is a compact quantum group. This construction is often useful when one deals with universal problems ([10] or [8]). We will use it since it is more precise than the direct $C^{*}$-construction for the definitions by generators and relations (no additional relations from $C^{*}$-norms).

When $A^{o}$ is a matrix Hopf $*$-algebra, i.e. is generated as a $*$-algebra by entries $u_{i j}$ of a matrix $u=\left(u_{i j}\right) \in M_{n}\left(A^{o}\right)$ such that $\Delta\left(u_{i j}\right)=\sum u_{i k} \otimes u_{k j}$ and $\varepsilon\left(u_{i j}\right)=\delta_{i j}$ (this condition is equivalent for $A^{o}$ to be a finite-type $*$-algebra), there is an easy way to see if $A^{o}$ is unitarizable: indeed $A^{o}$ is unitarizable if and only if there are matrices $F \in G L(\mathbb{C})$ and $G \in G L_{n}(\mathbb{C})$ such that the matrices $F u F^{-1}$ and $G \bar{u} G^{-1}$ are unitary; see 4 , 2.4. (S. Wang pointed out that the proof of this proposition in [4] is false. The conclusion is true, however.)

An action of a compact quantum group $A$ on a $C^{*}$-algebra $Z$ is a unital *homomorphism $\alpha: Z \longrightarrow Z \otimes A$ such that there is a dense sub-*-algebra $Z^{o}$ of $Z$ for which $\alpha$ restricts to a coaction $\alpha: Z^{o} \longrightarrow Z^{o} \otimes A^{o}$, i.e. $Z^{o}$ is a right $A^{\circ}$-comodule algebra. The category of compact quantum transformation groups of $Z$ is the category whose objects are compact quantum groups acting on $Z$ and whose morphisms are coaction preserving morphisms of compact quantum groups (see [8] for more details).

Definition 2.1 (8], 2.3). Let $Z$ be a $C^{*}$-algebra. The quantum automorphism group of $Z$ is a compact quantum group $A$ acting on $Z$ by $\alpha: Z \longrightarrow Z \otimes A$ and satisfying the following universal property: 
If $B$ is a compact quantum group acting on $Z$ by $\beta: Z \longrightarrow Z \otimes A$, there is a unique compact quantum group morphism $\pi: A \longrightarrow B$ such that $(1 \otimes \pi) \circ \alpha=\beta$.

When $X$ is a compact space, the quantum automorphism group of $X$ is the quantum automorphism group of the $C^{*}$-algebra $C(X)$.

Remarks. 1) When $Z$ is a finite-dimensional $C^{*}$-algebra, it is natural to think that the quantum automorphism group must be compact. For general $C^{*}$-algebras, the object defined above should be called the compact quantum automorphism group.

2 ) It is clear that the quantum automorphism group, if it exists, is unique up to isomorphism. It may not exist (see [8]).

Let $n \in \mathbb{N}^{*}$ and let $X_{n}$ be the space with $n$ points. The $C^{*}$-algebra $C\left(X_{n}\right)$ is the universal $*$-algebra with generators $\left(e_{i}\right)_{1 \leq i \leq n}$ and relations $e_{i}^{*}=e_{i} ; e_{i} e_{j}=\delta_{i j} e_{i}$ and $\sum_{i=1}^{n} e_{i}=1 ; 1 \leq i, j \leq n$. The following theorem gives the first example of a quantum automorphism group:

Theorem $2.2([8], 3.1)$. Let $A_{\text {aut }}^{o}\left(X_{n}\right)$ be the universal (complex) algebra with generators $\left(x_{i j}\right)_{1 \leq i, j \leq n}$ and with relations

$$
x_{i j} x_{i k}=\delta_{j k} x_{i j} ; x_{j i} x_{k i}=\delta_{j k} x_{j i} ; \sum_{l} x_{i l}=1=\sum_{l} x_{l i}, 1 \leq i, j, k \leq n .
$$

1) There is a Hopf *-algebra structure on $A_{\text {aut }}^{o}\left(X_{n}\right)$ defined by

$$
x_{i j}^{*}=x_{i j} ; \Delta\left(x_{i j}\right)=\sum_{k} x_{i k} \otimes x_{k j} ; \varepsilon\left(x_{i j}\right)=\delta_{i j} ; S\left(x_{i j}\right)=x_{j i}, 1 \leq i, j \leq n \text {. }
$$

Furthermore $A_{\text {aut }}^{o}\left(X_{n}\right)$ is a unitarizable Hopf *-algebra.

2) Let $A_{\text {aut }}\left(X_{n}\right)$ be the enveloping $C^{*}$-algebra of $A_{\text {aut }}^{o}\left(X_{n}\right)$. There is an action of $A_{\text {aut }}\left(X_{n}\right)$ on $C\left(X_{n}\right)$ defined by $\alpha\left(e_{i}\right)=\sum_{j} e_{j} \otimes x_{j i}$, and $A_{\text {aut }}\left(X_{n}\right)$ is the quantum automorphism group of the space $X_{n}$. The spectrum of $A_{\text {aut }}\left(X_{n}\right)$ is the symmetric group $S_{n}$.

The above quantum group is called the quantum permutation group on $n$ symbols. When $n \geq 4$ then $A_{a u t}\left(X_{n}\right)$ is a noncommutative and infinite-dimensional $C^{*}$-algebra.

Remark. Let $Z$ be a finite-dimensional noncommutative $C^{*}$-algebra. Wang shows in 8 that the quantum automorphism of $Z$ does not exist. However if $\psi=\operatorname{Tr}$ is a trace on $Z$, he shows the existence of the quantum automorphism group of the pair $(Z, \psi)$ (see [8] for the definition). We note here that this result can be obtained in the algebraic category of quantum transformation groups of $(Z, \psi)$, and the Hopf algebra representing the algebraic quantum automorphism group is a unitarizable Hopf $*$-algebra whose enveloping $C^{*}$-algebra is isomorphic with the quantum group described in theorem 5.1 of [8].

\section{QUANTUM AUTOMORPHISM GROUP OF A FINITE GRAPH}

In this paper a finite graph $\mathcal{G}=(V, E)$ is understood to be a pair $\mathcal{G}=(V, E)$ which consists of a finite set of vertices $V$ and a set of edges $E \subset V \times V$.

Let $s: E \rightarrow V$ (resp. $t: E \rightarrow V$ ) be the source map (resp. the target map). The source and target maps induce $*$-homomorphisms $s_{*}, t_{*}: C(V) \longrightarrow C(E)$.

An (usual) automorphism of a graph is a permutation of the vertices which preserves the set of edges. 
Definition 3.1. An action of a compact quantum group $A$ on a finite graph $\mathcal{G}=(V, E)$ consists of an action of $A$ on the set of vertices $\alpha: C(V) \longrightarrow C(V) \otimes A$ and an action of $A$ on the set of edges $\beta: C(E) \longrightarrow C(E) \otimes A$ such that the following diagram commutes:

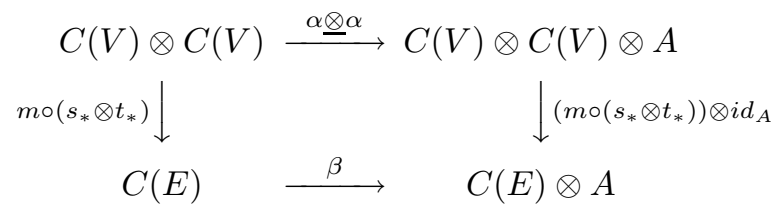

where $m: C(E) \otimes C(E) \rightarrow C(E)$ is the multiplication map of $C(E)$ and $\alpha \otimes \alpha$ is the tensor product of the coaction $\alpha$ by itself.

The quantum automorphism group of a finite graph $\mathcal{G}=(V, E)$ is a compact quantum group $A$ acting on $\mathcal{G}$ by $\alpha: C(V) \longrightarrow C(V) \otimes A$ and $\beta: C(E) \longrightarrow$ $C(E) \otimes A$, and satisfying the following universal property:

If $B$ is a compact quantum group acting on $\mathcal{G}$ by $\alpha^{\prime}: C(V) \longrightarrow C(V) \otimes B$ and $\beta^{\prime}: C(E) \longrightarrow C(E) \otimes B$, there is a unique morphism of compact quantum groups $\phi: A \longrightarrow B$ such that $(i d \otimes \phi) \circ \alpha=\alpha^{\prime}$ and $(i d \otimes \phi) \circ \beta=\beta^{\prime}$.

It is easy to see that this definition coincides with the usual one for compact groups. In the group case the action on the edges is entirely determined by the action on the vertices. This is also true in the quantum case (since the map $m \circ$ $\left(s_{*} \otimes t_{*}\right)$ is surjective).

Notation. Let $\mathcal{G}=(V, E)$ be a finite graph with $n$ vertices $V=\{1, \ldots, n\}$ and $m$ edges $E=\left\{\gamma_{1}, \ldots, \gamma_{m}\right\}$. Let $\left(e_{i}\right)_{1 \leq i \leq n}$ be the elements of $C(V)$ defined by $e_{i}(k)=\delta_{i k}, 1 \leq k \leq n$, and let $\left(f_{j}\right)_{1 \leq j \leq m}$ be the elements of $C(E)$ defined by $f_{j}\left(\gamma_{l}\right)=\delta_{j l}, 1 \leq l \leq m$.

Theorem 3.2. Let $\mathcal{G}=(V, E)$ be a finite graph with $n$ vertices and $m$ edges: $E=\left\{\gamma_{1}, \ldots, \gamma_{m}\right\}$. Let $A_{a u t}^{o}(\mathcal{G})$ be the universal complex algebra with generators $\left(X_{i j}\right)_{1 \leq i, j \leq n}$ and relations

$$
X_{i j} X_{i k}=\delta_{j k} X_{i j} ; X_{j i} X_{k i}=\delta_{j k} X_{j i} ; \sum_{l=1}^{n} X_{i l}=1=\sum_{l=1}^{n} X_{l i}, 1 \leq i, j, k \leq n,
$$

$$
\begin{gathered}
X_{s\left(\gamma_{j}\right) i} X_{t\left(\gamma_{j}\right) k}=X_{t\left(\gamma_{j}\right) k} X_{s\left(\gamma_{j}\right) i}=0, \\
X_{i s\left(\gamma_{j}\right)} X_{k t\left(\gamma_{j}\right)}=X_{k t\left(\gamma_{j}\right)} X_{i s\left(\gamma_{j}\right)}=0 \quad, \quad \gamma_{j} \in E, \quad(i, k) \notin E, \\
X_{s\left(\gamma_{j}\right) s\left(\gamma_{l}\right)} X_{t\left(\gamma_{j}\right) t\left(\gamma_{l}\right)}=X_{t\left(\gamma_{j}\right) t\left(\gamma_{l}\right)} X_{s\left(\gamma_{j}\right) s\left(\gamma_{l}\right)} \quad, \gamma_{j}, \gamma_{l} \in E, \\
\sum_{l=1}^{m} X_{s\left(\gamma_{l}\right) s\left(\gamma_{j}\right)} X_{t\left(\gamma_{l}\right) t\left(\gamma_{j}\right)}=1=\sum_{l=1}^{m} X_{s\left(\gamma_{j}\right) s\left(\gamma_{l}\right)} X_{t\left(\gamma_{j}\right) t\left(\gamma_{l}\right)} \quad, \quad \gamma_{j} \in E .
\end{gathered}
$$

1) There is a Hopf *-algebra structure on $A_{\text {aut }}^{o}(\mathcal{G})$ defined by

$X_{i j}^{*}=X_{i j} ; \Delta\left(X_{i j}\right)=\sum_{k=1}^{n} X_{i k} \otimes X_{k j} ; \varepsilon\left(X_{i j}\right)=\delta_{i j} ; S\left(X_{i j}\right)=X_{j i} ; 1 \leq i, j \leq n$, and $A_{\text {aut }}^{o}(\mathcal{G})$ is a unitarizable Hopf *-algebra. 
2) Let $A_{\text {aut }}(\mathcal{G})$ be the enveloping $C^{*}$-algebra of $A_{\text {aut }}^{o}(\mathcal{G})$. The formulas

$$
\begin{gathered}
\alpha\left(e_{i}\right)=\sum_{k=1}^{n} e_{k} \otimes X_{k i} \quad, \quad 1 \leq i \leq n, \\
\beta\left(f_{j}\right)=\sum_{l=1}^{m} f_{l} \otimes X_{s\left(\gamma_{l}\right) s\left(\gamma_{j}\right)} X_{t\left(\gamma_{l}\right) t\left(\gamma_{j}\right)} \quad, \quad 1 \leq j \leq m,
\end{gathered}
$$

define an action of $A_{\text {aut }}(\mathcal{G})$ on $\mathcal{G}$, and $A_{\text {aut }}(\mathcal{G})$ is the quantum automorphism group of $\mathcal{G}$. The spectrum of $A_{\text {aut }}(\mathcal{G})$ is the usual automorphism group of $\mathcal{G}$.

Proof. Let $I$ be the two-sided ideal of $A_{\text {aut }}^{o}\left(X_{n}\right)$ generated by relations (3.2), (3.3) and (3.4). Obviously we have $A_{\text {aut }}^{o}(\mathcal{G}) \cong A_{\text {aut }}^{o}\left(X_{n}\right) / I$. It is easy to see that $I$ is a *-ideal and hence $A_{\text {aut }}^{o}(\mathcal{G})$ is a *-algebra. We must prove that the maps $\varepsilon, \Delta$ and $S$ of the theorem are well defined. It is easy to check that the character $\varepsilon$ and the anti-homomorphism $S$ are well defined. Let $\gamma_{j} \in E$ and $(i, k) \in V \times V$ be such that $(i, k) \notin E$. We have

$$
\begin{aligned}
\Delta\left(X_{s\left(\gamma_{j}\right) i} X_{t\left(\gamma_{j}\right) k}\right) & =\sum_{p, q}^{n} X_{s\left(\gamma_{j}\right) p} X_{t\left(\gamma_{j}\right) q} \otimes X_{p i} X_{q k} \\
& \left.=\sum_{p, q(p, q) \in E} X_{s\left(\gamma_{j}\right) p} X_{t\left(\gamma_{j}\right) q} \otimes X_{p i} X_{q k} \quad \text { (by } \quad(3.2)\right) \\
& =\sum_{l=1}^{m} X_{s\left(\gamma_{j}\right) s\left(\gamma_{l}\right)} X_{t\left(\gamma_{j}\right) t\left(\gamma_{l}\right)} \otimes X_{s\left(\gamma_{l}\right) i} X_{t\left(\gamma_{l}\right) k}=0 \quad \text { (by }
\end{aligned}
$$

In the same way we have

$$
\Delta\left(X_{t\left(\gamma_{j}\right) k} X_{s\left(\gamma_{j}\right) i}\right)=0=\Delta\left(X_{i s\left(\gamma_{j}\right)} X_{k t\left(\gamma_{j}\right)}\right)=\Delta\left(X_{k t\left(\gamma_{j}\right)} X_{i s\left(\gamma_{j}\right)}\right) .
$$

Let $\gamma_{j}, \gamma_{l} \in E$. We have

$$
\begin{aligned}
\Delta\left(X_{s\left(\gamma_{j}\right) s\left(\gamma_{l}\right)} X_{t\left(\gamma_{j}\right) t\left(\gamma_{l}\right)}\right) & =\sum_{i, k}^{n} X_{s\left(\gamma_{j}\right) i} X_{t\left(\gamma_{j}\right) k} \otimes X_{i s\left(\gamma_{l}\right)} X_{k t\left(\gamma_{l}\right)} \\
(\text { by }(3.2)) & =\sum_{l^{\prime}=1}^{m} X_{s\left(\gamma_{j}\right) s\left(\gamma_{l^{\prime}}\right)} X_{t\left(\gamma_{j}\right) t\left(\gamma_{l^{\prime}}\right)} \otimes X_{s\left(\gamma_{l^{\prime}}\right) s\left(\gamma_{l}\right)} X_{t\left(\gamma_{l^{\prime}}\right) t\left(\gamma_{l}\right)} \\
(\text { by }(3.3)) & =\sum_{l^{\prime}=1}^{m} X_{t\left(\gamma_{j}\right) t\left(\gamma_{l^{\prime}}\right)} X_{s\left(\gamma_{j}\right) s\left(\gamma_{l^{\prime}}\right)} \otimes X_{t\left(\gamma_{l^{\prime}}\right) t\left(\gamma_{l}\right)} X_{s\left(\gamma_{l^{\prime}}\right) s\left(\gamma_{l}\right)} \\
(\text { by (3.2)) } & =\sum_{i, k}^{n} X_{t\left(\gamma_{j}\right) i} X_{s\left(\gamma_{j}\right) k} \otimes X_{i t\left(\gamma_{l}\right)} X_{k s\left(\gamma_{l}\right)} \\
& =\Delta\left(X_{t\left(\gamma_{j}\right) t\left(\gamma_{l}\right)} X_{s\left(\gamma_{j}\right) s\left(\gamma_{l}\right)}\right) .
\end{aligned}
$$

Now let $\gamma_{j} \in V$. We have

$$
\begin{aligned}
\Delta\left(\sum_{l=1}^{m} X_{s\left(\gamma_{l}\right) s\left(\gamma_{j}\right)} X_{t\left(\gamma_{l}\right) t\left(\gamma_{j}\right)}\right) & =\sum_{l=1}^{m} \sum_{k, i}^{n} X_{s\left(\gamma_{l}\right) i} X_{t\left(\gamma_{l}\right) k} \otimes X_{i s\left(\gamma_{j}\right)} X_{k t\left(\gamma_{j}\right)} \\
(\text { by }(3.2)) & =\sum_{l, l^{\prime}}^{m} X_{s\left(\gamma_{l}\right) s\left(\gamma_{l^{\prime}}\right)} X_{t\left(\gamma_{l}\right) t\left(\gamma_{l^{\prime}}\right)} \otimes X_{s\left(\gamma_{l^{\prime}}\right) s\left(\gamma_{j}\right)} X_{t\left(\gamma_{l^{\prime}}\right) t\left(\gamma_{j}\right)} \\
& =1 \otimes 1=\Delta(1) \quad(\text { by }(3.4)) .
\end{aligned}
$$


In the same way $\Delta\left(\sum_{l=1}^{m} X_{s\left(\gamma_{j}\right) s\left(\gamma_{l}\right)} X_{t\left(\gamma_{j}\right) t\left(\gamma_{l}\right)}\right)=\Delta(1)$. Therefore $\Delta$ is a well defined algebra morphism and in this way $A_{\text {aut }}^{o}(\mathcal{G})$ is a bialgebra. It is clear that the antihomomorphism $S$ is an antipode for this bialgebra and hence $A_{\text {aut }}^{o}(\mathcal{G})$ is a Hopf $*$-algebra which is clearly unitarizable. There is an obvious surjective Hopf *-algebra morphism $\pi: A_{\text {aut }}^{o}\left(X_{n}\right) \longrightarrow A_{\text {aut }}^{o}(\mathcal{G})$ defined by $\pi\left(x_{i j}\right)=X_{i j}$.

2) By relations (3.1) and Theorem 2.2 we have an action $\alpha: C(V) \longrightarrow C(V) \otimes$ $A_{\text {aut }}(\mathcal{G})$ as defined in the theorem. Let $\gamma_{j}, \gamma_{l}$ and $\gamma_{j^{\prime}} \in E$. By (3.3) we have

$$
\left(X_{s\left(\gamma_{l}\right) s\left(\gamma_{j}\right)} X_{t\left(\gamma_{l}\right) t\left(\gamma_{j}\right)}\right)^{*}=X_{s\left(\gamma_{l}\right) s\left(\gamma_{j}\right)} X_{t\left(\gamma_{l}\right) t\left(\gamma_{j}\right)}
$$

and by (3.1), (3.2) and (3.3) we have

$$
X_{s\left(\gamma_{l}\right) s\left(\gamma_{j}\right)} X_{t\left(\gamma_{l}\right) t\left(\gamma_{j}\right)} X_{s\left(\gamma_{l}\right) s\left(\gamma_{j^{\prime}}\right)} X_{t\left(\gamma_{l}\right) t\left(\gamma_{j^{\prime}}\right)}=\delta_{j j^{\prime}} X_{s\left(\gamma_{l}\right) s\left(\gamma_{j}\right)} X_{t\left(\gamma_{l}\right) t\left(\gamma_{j}\right)} .
$$

Finally using (3.4) we see that there is a $*$-homomorphism $\beta: C(E) \longrightarrow C(E) \otimes$ $A_{\text {aut }}(\mathcal{G})$ as defined in the theorem and $\beta$ is coassociative (see the calculations in 1$)$ ). Let us check that the diagram ( $\star$ ) commutes. Let $i, k \in V$. We have $\alpha \underline{\otimes} \alpha\left(e_{i} \otimes e_{k}\right)=$ $\sum_{p, q}^{n} e_{p} \otimes e_{q} \otimes X_{p i} X_{q k}$. We also have $s_{*}\left(e_{i}\right)=\sum_{l, s\left(\gamma_{l}\right)=i} f_{l}$ and $t_{*}\left(e_{k}\right)=\sum_{l, t\left(\gamma_{l}\right)=k} f_{l}$. Hence

$$
\begin{aligned}
\left(\left(m \circ\left(s_{*} \otimes t_{*}\right)\right) \otimes i d\right) \circ(\alpha \underline{\otimes} \alpha) & =\sum_{p, q}^{n} \sum_{l,(p, q)=\gamma_{l}} f_{l} \otimes X_{p i} X_{q k} \\
& =\sum_{j=1}^{m} f_{j} \otimes X_{s\left(\gamma_{j}\right) i} X_{t\left(\gamma_{j}\right) k} .
\end{aligned}
$$

On the other hand

$$
\begin{aligned}
\beta \circ m \circ\left(s_{*} \otimes t_{*}\right)\left(e_{i} \otimes e_{k}\right) & =\sum_{l, \gamma_{l}=(i, k)} \sum_{j=1}^{m} f_{j} \otimes X_{s\left(\gamma_{j}\right) s\left(\gamma_{l}\right)} X_{t\left(\gamma_{j}\right) t\left(\gamma_{l}\right)} \\
& =\sum_{j=1}^{m} f_{j} \otimes X_{s\left(\gamma_{j}\right) i} X_{t\left(\gamma_{j}\right) k} .
\end{aligned}
$$

In this way we have defined an action of $A_{\text {aut }}(\mathcal{G})$ on the graph $\mathcal{G}$.

Let $B$ be a compact quantum group acting on $\mathcal{G}$ by $\alpha^{\prime}: C(V) \longrightarrow C(V) \otimes B$ and $\beta^{\prime}: C(E) \longrightarrow C(E) \otimes B$. There are elements $\left(a_{i j}\right)_{1 \leq i, j \leq n}$ of $B$ such that $\alpha^{\prime}\left(e_{i}\right)=\sum_{j} e_{j} \otimes a_{j i}$ and by Theorem 2.2 these elements satisfy relations (3.1) (the elements $a_{i j}$ belong to the algebra of representative functions $B^{o}$ ). There are also elements $\left(b_{j l}\right)_{1 \leq j, l \leq m}$ of $B^{o}$ such that $\beta^{\prime}\left(f_{j}\right)=\sum_{l} f_{l} \otimes b_{l j}$ and Theorem 2.2 ensures that they satisfy the same relations as (3.1). By the commutativity of the diagram $(\star)$, we have $b_{l j}=a_{s\left(\gamma_{l}\right) s\left(\gamma_{j}\right)} a_{t\left(\gamma_{l}\right) t\left(\gamma_{j}\right)}$ and hence there is a surjective morphism of compact quantum groups $\phi_{0}: A_{\text {aut }}\left(X_{n}\right) \longrightarrow B$ defined by $\phi_{0}\left(x_{i k}\right)=a_{i k}$. It remains to check that the $a_{i k}$ 's satisfy relations (3.2), (3.3) and (3.4). We have $b_{l j}^{*}=b_{l j}$ and hence

$$
a_{t\left(\gamma_{l}\right) t\left(\gamma_{j}\right)} a_{s\left(\gamma_{l}\right) s\left(\gamma_{j}\right)}=a_{s\left(\gamma_{l}\right) s\left(\gamma_{j}\right)} a_{t\left(\gamma_{l}\right) t\left(\gamma_{j}\right)} \quad, \quad \gamma_{j}, \gamma_{l} \in E,
$$

and relations (3.3) hold. We have $\sum_{l=1}^{m} b_{l j}=1=\sum_{l=1}^{m} b_{j l}, \forall \gamma_{j} \in E$ and hence relations (3.4) are satisfied. Using once again the commutativity of the diagram $(\star)$, we get $a_{s\left(\gamma_{j}\right) i} a_{t\left(\gamma_{j}\right) k}=0$ whenever $(i, k) \notin E$. Using the involution and the antipode of $B^{o}$, it is easy to see that the other relations in (3.2) are fulfilled. Thus we have a morphism of quantum groups $\phi: A_{\text {aut }}(\mathcal{G}) \longrightarrow B$ such that $(i d \otimes \phi) \circ \alpha=\alpha^{\prime}$ 
and $(i d \otimes \phi) \circ \beta=\beta^{\prime}$ : this morphism is obviously unique and $A_{a u t}(\mathcal{G})$ is the quantum automorphism group of $\mathcal{G}$. The last statement follows immediately from the universal property of $A_{\text {aut }}(\mathcal{G})$.

Let us take a look at some examples. First let us consider the graph $\mathcal{G}=(V, E)$ with $n$ vertices and $E=\emptyset$. It is obvious that $A_{a u t}(\mathcal{G})$ is the quantum permutation group $A_{\text {aut }}\left(X_{n}\right)$. Less trivially, let us consider the graph $\mathcal{G}=(V, E)$ with $n$ vertices $V=\{1, \ldots, n\}$ and $E=\{(i, i), i \in E\}$. Then it is easy to check that relations (3.2), (3.3) and (3.4) all follow from (3.1) and hence $A_{\text {aut }}(\mathcal{G})=A_{\text {aut }}\left(X_{n}\right)$.

Now let us consider the complete graph $\mathcal{G}=(V, E)$ with $n$ vertices and $E=$ $V \times V$. Then relations (3.3) imply that $A_{a u t}(\mathcal{G})$ is a commutative $C^{*}$-algebra. Since the spectrum of $A_{\text {aut }}(\mathcal{G})$ is the usual automorphism group of $\mathcal{G}$ (in this case the symmetric group $\left.S_{n}\right)$, we have $A_{\text {aut }}(\mathcal{G}) \cong C\left(S_{n}\right)$. Hence the quantum automorphism group and the usual one coincide in this example.

These simple examples show that the quantum automorphism group is a stronger invariant for finite graphs than the usual automorphism group.

Another elementary example is the following one. Let us consider the polygonal graph $\mathcal{G}$ with $n$ vertices $V=\{1, \ldots, n\}$ and $E=\left\{\gamma_{1}, \ldots, \gamma_{n}\right\}$ where $\gamma_{i}=(i, i+1)$, $1 \leq i \leq n-1$ and $\gamma_{n}=(n, 1)$. It is easily seen that $A_{\text {aut }}(\mathcal{G}) \cong C(\mathbb{Z} / n \mathbb{Z})$, the algebra of functions on the cyclic group $\mathbb{Z} / n \mathbb{Z}$.

We now examine a less trivial example:

Proposition 3.3. Let $\mathcal{G}=(V, E)$ be the graph with 4 vertices $V=\{1,2,3,4\}$ and 4 edges $E=\left\{\gamma_{1}, \gamma_{2}, \gamma_{3}, \gamma_{4}\right\}$ where $\gamma_{1}=(1,2), \gamma_{2}=(2,1), \gamma_{3}=(3,4)$ and $\gamma_{4}=(4,3)$. Then $A_{\text {aut }}(\mathcal{G})$ is a noncommutative infinite-dimensional $C^{*}$-algebra whose spectrum is the dihedral group $D_{4}$.

The quantum group above will be called the quantum dihedral group $D_{4}$.

Proof. It is easily seen that the usual automorphism group of the above graph is a finite group with 8 elements which is the dihedral group $D_{4}$ and hence by Theorem 3.2 the spectrum of $A_{\text {aut }}(\mathcal{G})$ is the group $D_{4}$.

Let us now describe the $*$-algebra $A_{\text {aut }}^{o}(\mathcal{G})$. First let us translate relations $(3.2)$.

We get the following relations $(3.2)^{\prime}$ :

$$
\begin{aligned}
& X_{11} X_{23}=X_{23} X_{11}=0=X_{32} X_{11}=X_{11} X_{32}, \\
& X_{11} X_{24}=X_{24} X_{11}=0=X_{42} X_{11}=X_{11} X_{42}, \\
& X_{12} X_{23}=X_{23} X_{12}=0=X_{32} X_{21}=X_{21} X_{32}, \\
& X_{12} X_{24}=X_{24} X_{12}=0=X_{42} X_{21}=X_{21} X_{42}, \\
& X_{13} X_{21}=X_{21} X_{13}=0=X_{31} X_{12}=X_{12} X_{31}, \\
& X_{13} X_{22}=X_{22} X_{13}=0=X_{31} X_{22}=X_{22} X_{31}, \\
& X_{14} X_{22}=X_{22} X_{14}=0=X_{41} X_{22}=X_{22} X_{41}, \\
& X_{14} X_{21}=X_{21} X_{14}=0=X_{12} X_{41}=X_{41} X_{12}, \\
& X_{31} X_{43}=X_{43} X_{31}=0=X_{13} X_{34}=X_{34} X_{13}, \\
& X_{31} X_{44}=X_{44} X_{31}=0=X_{13} X_{44}=X_{44} X_{13}, \\
& X_{32} X_{43}=X_{43} X_{32}=0=X_{23} X_{34}=X_{34} X_{23}, \\
& X_{32} X_{44}=X_{44} X_{32}=0=X_{23} X_{44}=X_{44} X_{23}, \\
& X_{33} X_{41}=X_{41} X_{33}=0=X_{33} X_{14}=X_{14} X_{33}, \\
& X_{33} X_{42}=X_{42} X_{33}=0=X_{33} X_{24}=X_{24} X_{33}, \\
& X_{34} X_{42}=X_{42} X_{34}=0=X_{43} X_{24}=X_{24} X_{43}, \\
& X_{34} X_{41}=X_{41} X_{34}=0=X_{14} X_{43}=X_{43} X_{14} .
\end{aligned}
$$


Relations (3.3) are translated in the following relations (3.3)':

$$
\begin{array}{lll}
X_{11} X_{22}=X_{22} X_{11} & , & X_{31} X_{42}=X_{42} X_{31}, \\
X_{12} X_{21}=X_{21} X_{12} & , & X_{32} X_{41}=X_{41} X_{32}, \\
X_{13} X_{24}=X_{24} X_{13} & , & X_{33} X_{44}=X_{44} X_{33}, \\
X_{14} X_{23}=X_{23} X_{14} & , & X_{34} X_{43}=X_{43} X_{34}
\end{array}
$$

Relations (3.4) become relations (3.4)':

$X_{11} X_{22}+X_{21} X_{12}+X_{31} X_{42}+X_{41} X_{32}=1=X_{11} X_{22}+X_{12} X_{21}+X_{13} X_{24}+X_{14} X_{23}$, $X_{12} X_{21}+X_{22} X_{11}+X_{32} X_{41}+X_{42} X_{31}=1=X_{21} X_{12}+X_{22} X_{11}+X_{23} X_{14}+X_{24} X_{13}$, $X_{13} X_{24}+X_{23} X_{14}+X_{33} X_{44}+X_{43} X_{34}=1=X_{31} X_{42}+X_{32} X_{41}+X_{33} X_{44}+X_{34} X_{43}$, $X_{14} X_{23}+X_{24} X_{13}+X_{34} X_{43}+X_{44} X_{33}=1=X_{41} X_{32}+X_{42} X_{31}+X_{43} X_{34}+X_{44} X_{33}$. Combining (3.1), $(3.2)^{\prime},(3.3)^{\prime},(3.4)^{\prime}$ we get the following useful relations:

$$
\begin{aligned}
& X_{11}=X_{22}=X_{11} X_{22}=X_{22} X_{11} \quad, \quad X_{12}=X_{21}=X_{12} X_{21}=X_{21} X_{12}, \\
& X_{13}=X_{24}=X_{13} X_{24}=X_{24} X_{13} \quad, \quad X_{14}=X_{23}=X_{14} X_{23}=X_{23} X_{14}, \\
& X_{31}=X_{42}=X_{31} X_{42}=X_{42} X_{31} \quad, \quad X_{32}=X_{41}=X_{32} X_{41}=X_{41} X_{32}, \\
& X_{33}=X_{44}=X_{33} X_{44}=X_{44} X_{33} \quad, \quad X_{34}=X_{43}=X_{34} X_{43}=X_{43} X_{34} .
\end{aligned}
$$

We are now able to describe the algebra $A_{\text {aut }}^{o}(\mathcal{G})$ in a simpler way. Let $B^{o}$ be the universal $*$-algebra with generators $\left(y_{i}\right)_{1 \leq i \leq 8}$ and relations

$$
\begin{array}{r}
y_{i}^{*}=y_{i}^{2}=y_{i}, 1 \leq i \leq 8 \quad ; \quad y_{1} y_{i}=0=y_{i} y_{1}, 2 \leq i \leq 6 \quad ; \\
y_{2} y_{i}=0=y_{i} y_{2}, 3 \leq i \leq 6 \quad ; \quad y_{3} y_{i}=0=y_{i} y_{3}, i \in\{4,7,8\} \quad ; \\
y_{4} y_{i}=0=y_{i} y_{4}, i \in\{7,8\} \quad ; \quad y_{5} y_{i}=0=y_{i} y_{5}, i \in\{6,7,8\} \quad ; \\
y_{6} y_{i}=0=y_{i} y_{6}, i \in\{7,8\} \quad ; \quad y_{7} y_{8}=0=y_{8} y_{7} \quad ; \\
y_{1}+y_{2}+y_{3}+y_{4}=y_{1}+y_{2}+y_{5}+y_{6}=1=y_{3}+y_{4}+y_{7}+y_{8}=y_{5}+y_{6}+y_{7}+y_{8} .
\end{array}
$$

The reader will easily check that there is a $*$-homomorphism $\varphi: B^{o} \longrightarrow A_{\text {aut }}^{o}(\mathcal{G})$ defined by $\varphi\left(y_{1}\right)=X_{11}, \varphi\left(y_{2}\right)=X_{12}, \varphi\left(y_{3}\right)=X_{13}, \varphi\left(y_{4}\right)=X_{14}, \varphi\left(y_{5}\right)=X_{31}$, $\varphi\left(y_{6}\right)=X_{32}, \varphi\left(y_{7}\right)=X_{33}, \varphi\left(y_{8}\right)=X_{34}$.

In the same way there is a $*$-homomorphism $\psi: A_{\text {aut }}^{o}(\mathcal{G}) \longrightarrow B^{o}$ defined by $\psi\left(X_{11}\right)=\psi\left(X_{22}\right)=y_{1}, \psi\left(X_{12}\right)=\psi\left(X_{21}\right)=y_{2}, \psi\left(X_{13}\right)=\psi\left(X_{24}\right)=y_{3}, \psi\left(X_{14}\right)=$ $\psi\left(X_{23}\right)=y_{4}, \psi\left(X_{31}\right)=\psi\left(X_{42}\right)=y_{5}, \psi\left(X_{32}\right)=\psi\left(X_{41}\right)=y_{6}, \psi\left(X_{33}\right)=\psi\left(X_{44}\right)=$ $y_{7}, \psi\left(X_{34}\right)=\psi\left(X_{43}\right)=y_{8}$.

We have $\varphi \circ \psi=i d$ and $\psi \circ \varphi=i d$ and therefore the $*$-algebras $B^{\circ}$ and $A_{\text {aut }}^{o}(\mathcal{G})$ are isomorphic.

Let us now show that $B^{o}$ is noncommutative and infinite-dimensional. There is a representation $\pi: B^{o} \longrightarrow M_{2}(\mathbb{C})$ defined by

$$
\pi\left(y_{1}\right)=\left(\begin{array}{ll}
1 & 0 \\
0 & 0
\end{array}\right), \pi\left(y_{2}\right)=\left(\begin{array}{ll}
0 & 0 \\
0 & 1
\end{array}\right), \pi\left(y_{7}\right)=\left(\begin{array}{ll}
1 & 0 \\
1 & 0
\end{array}\right), \pi\left(y_{8}\right)=\left(\begin{array}{cc}
0 & 0 \\
-1 & 1
\end{array}\right)
$$

and $\pi\left(y_{3}\right)=\pi\left(y_{4}\right)=\pi\left(y_{5}\right)=\pi\left(y_{6}\right)=0$. We have $\pi\left(y_{1} y_{7}\right)=\pi\left(y_{1}\right)$ while $\pi\left(y_{7} y_{1}\right)=$ $\pi\left(y_{7}\right): B^{o}$ is not commutative.

Let us suppose that $B^{o}$ is finite-dimensional. Then $B^{o}$ would be a finitedimensional $C^{*}$-algebra since $B^{o} \cong A_{\text {aut }}^{o}(\mathcal{G})$. But $\pi\left(B^{o}\right)$ is contained in the algebra of lower-triangular matrices. This means that the representation $\pi$ is not semisimple: we have a contradiction. Hence $A_{\text {aut }}(\mathcal{G}) \cong C^{*}\left(B^{o}\right)$ is infinite-dimensional.

Proposition 3.3 gives a concrete example of a non-trivial quantum subgroup (i.e. noncommutative and noncocommutative) of $A_{\text {aut }}\left(X_{n}\right)$. It is expected that the construction described in [1] furnishes many other examples of this kind. 


\section{ACKNOWLEDGEMENTS}

The author wishes to thank G. Laffaille who read the manuscript.

\section{REFERENCES}

[1] T. BAnicA, Quantum groups acting on $N$ points, complex Hadamard matrices, and a construction of subfactors, Preprint OA/9806054.

[2] T. BAnICA, Symmetries of a generic coaction, Math. Ann. 314, 763-780, 1999. MR 2001g:46146

[3] A. Connes, Noncommutative geometry. London: Academic Press, 1994. MR 95j:46063

[4] M.S. Dijkhuizen, T.H. Koornwinder, CQG algebras: a direct algebraic approach to compact quantum groups, Lett. Math. Phys. 32, 315-330, 1994. MR 95m:16029

[5] Y. Manin, Quantum groups and noncommutative geometry. Publications du CRM 1561, Univ. de Montréal, 1988. MR 91e:17001

[6] A. Van Daele, The Haar measure on a compact quantum group, Proc. Amer. Math. Soc. 123 (10), 3125-3128, 1995. MR 95m:46097

[7] S. WAng, Free products of compact quantum groups, Comm. Math. Phys. 167, 671-692, 1995. MR 95k:46104

[8] S. WANG, Quantum symmetry groups of finite spaces, Comm. Math. Phys. 195, 195-211, 1998. MR 99h:58014

[9] S.L. Woronowicz, Compact matrix pseudogroups, Comm. Math. Phys. 111, 613-665, 1987. MR 88m:46079

[10] S.L. Woronowicz, Tannaka-Krein duality for compact matrix pseudogroups. Twisted $S U(N)$ groups, Invent. Math. 93, 35-76, 1988. MR 90e:22033

[11] S.L. Woronowicz, Compact quantum groups, in "Symétries quantiques" (Les Houches, 1995), North Holland, Amsterdam, 1998, 845-884. MR 99m:46164

Département des Sciences Mathématiques, case 051, Université Montpellier II, Place Eugène Bataillon, 34095 Montpellier Cedex 5, France

Current address: Département de Mathématiques, Université de Pau et de Pays de l'Adour, Avenue de l'université, 64000 Pau, France

E-mail address: Julien.Bichon@univ-pau.fr 